\title{
Relationship between pre-sarcopenia, sarcopenia and bone mineral density in elderly men
}

Fernando Borges Pereira' ${ }^{1}$, André Ferreira Leite ${ }^{2}$, Ana Patrícia de Paula ${ }^{3}$

\begin{abstract}
Objective: Analyze the influence of sarcopenia in bone health of elderly men. Subjects and methods: This cross-sectional study evaluated 198 men aged over 60 years. Body composition was measured by dual energy X-ray absorptiometry. The BMD was measured the femoral neck, total hip, lumbar spine and $33 \%$ radius. The diagnosis of abnormal BMD was defined for men who presented densitometric diagnosis of osteopenia or osteoporosis defined by T-score of femoral neck, total hip and lumbar spine. The pre-sarcopenia and sarcopenia were defined according to the European Working Group on Sarcopenia in Older People. Results: The group diagnosed with normal BMD, compared to the group of abnormal BMD, have significantly higher body weight, body mass index, grip strength, lean mass, fat mass, and relative appendicular skeletal muscle mass (RASM). However, after multiple linear regression analysis, we found that only the RASM, lean mass, and handgrip strength in the dominant hand influenced the variability of the BMD after adjustment for age and weight. Regression analyzes showed a positive association between greater appendicular lean mass and a smaller number of elderly patients with abnormal BMD diagnostic. The regression analyzes showed that elderly men diagnosed with pre-sarcopenia and sarcopenia had more abnormal BMD than non-sarcopenic elderly men. Conclusion: We concluded that pre-sarcopenia and sarcopenia were associated with abnormal BMD. The lean mass, compared to fat mass, has a greater positive influence on the BMD of elderly men. This result suggests the importance of the increase in lean mass for the bone health of elderly men. Arch Endocrinol Metab. 2015;59(1):59-65
\end{abstract}

Keywords

Sarcopenia; osteoporosis; bone mineral density; muscle mass; elderly
1 Faculdade de Ciências da Saúde, Universidade de Brasília (UnB), Brasilia, DF, Brazil ${ }^{2}$ Faculdade de Ciências da Saúde, UnB, Distrito Federal, Brasília, DF, Brazil ${ }^{3}$ Hospital de Base de Brasilia, Brasília, DF, Brazil

Correspondence to: Fernando Borges Pereira QE 15, conjunto $M$, casa 19, Guará 2 71050-131 - Brasília, DF, Brazil feborgesprofessor@gmail.com

Received on June/10/2014 Accepted on Oct/10/2014

DOI: 10.1590/2359-3997000000011

\section{INTRODUCTION}

$\mathrm{O}$ steoporosis is a major public health problem in the male population (1). Total costs including prevalent fractures are more than $\$ 19$ billion in the United States. Men account for $29 \%$ of fractures and $25 \%$ of costs (2).

Sarcopenia has emerged as an important risk factor for osteoporosis $(3,4)$. In addition to increasing falls risk, sarcopenia might also decrease bone strength by reducing mechanical loading to the skeleton. Reduction of mechanical stimulation could result from decreased maximal force that weaker muscles produce and/or less time that the skeleton is loaded due to relative immobility, and thus bone formation is reduced (5-7). The evidence for this relationship comes primarily from observational epidemiological studies in women $(5,7)$. However, the available data regarding this association are inconsistent, mainly due to a lack of definition in the diagnosis of sarcopenia $(8,9)$.

Recently, the European Working Group on Sarcopenia in Older People (EWGSOP) suggested criteria and sub-classifications for a definition of sarcopenia, which are based not only on the assessment of the reduction in fat-free mass but also on the assessment of loss of muscle strength and physical performance (8).

Few studies have evaluated the influence of sarcopenia as diagnosed by the setting EWGSOP in bone mineral density (BMD) in older men. Elucidating this relationship will enable the development of more effective strategies for the prevention of osteoporosis in the male population.

The aim of this study is analyze the influence of sarcopenia in bone health of elderly men using the more stringent EWGSOP definition of sarcopenia. 


\section{MATERIALS AND METHODS}

\section{Subjects}

The present study included healthy men aged over or equal to 60 years from a community in São SebastiãoFederal District, Brazil. Subjects institutionalized or in active bone therapies (corticosteroids, bisphosphonates, parathyroid hormone, calcium and vitamin D) were excluded from the study. Subjects using medication or having a disease like rheumatoid arthritis, inflammatory myopathy, prolonged immobilization, or cancer were also excluded.

We tried to contact five hundred and twenty four elderly men with a history of vaccination in the São Sebastião's health center (Federal District, Brazil). Only 265 elderly men with a valid telephone were found. Seventeen men refused to participate in the study and five had died. Two hundred and forty three men were initially evaluated, 226 met the inclusion and exclusion criteria, and 198 underwent bone densitometry by DXA for assessment of bone mineral density and body composition and muscle strength evaluation.

The 198 elderly men were evaluated for the presence of clinical risk factors for osteoporotic fracture like eating habits, smoking, alcohol consumption, associated diseases, medications, and family and personal history of fragility fractures. The patients underwent all reviews in less than 60 days. All participants agreed and signed the consent form. The Research Ethics Committee of the Faculty of Health Sciences, University of Brasilia, approved the study.

A scale with stadiometer Filizol ${ }^{\circledR}$ assessed weight and height. Body mass index (BMI) was calculated dividing weight in kilograms by height in meters squared.

\section{Dual energy X-ray absorptiometry (DXA) for bone parameters and body composition analysis}

Bone densitometry by DXA was performed at the University Hospital of Brasilia on equipment branded $\mathrm{Lu}-$ nar DPX NT (GE Medical Systems, Waukesha, Wisconsin, USA). The performance of the densitometries of the whole body, lumbar spine, femoral neck, total hip, and 33\% radius non-dominant followed the manufacturer's instructions and were performed by the same operator. The results of the bone densitometry were expressed in $\mathrm{g} / \mathrm{cm}^{2}$ and T-score, calculated by the device itself, and analyzed according to the criteria of the World Health Organization (10). A whole body DXA scan was also performed to measure total lean mass, lean mass of arms and legs, and fat mass using fan beam technology. The analyses were performed by a single doctor with the Encore 2005 program, version 9.1, according to the manufacturer's standards and respecting the standards of The International Society for Clinical Densitometry (ISCD). During the measurements, all patients wore light cotton clothes without underwire bras, metal buttons, zippers, metallic paint, or threads. Precision values were calculated based on the short-term, in vivo precision study in which duplicate measurements were performed with repositioning between each scan in the 30 elderly men. A daily quality assurance scan was conducted by scanning an aluminum spine phantom according to the manufacturer's instructions.

The coefficients of variation (CVs) of the lumbar spine $\mathrm{BMD}$, the femoral neck BMD, total hip BMD, lean mass and fat mass measurements were $1 \%, 1.6 \%$, $1.8 \%, 0.74 \%$ and $1.5 \%$, respectively.

\section{Muscle components}

Appendicular lean mass (aLM $(\mathrm{kg})$ ) was determined by the sum of arms and legs lean mass (11).

The relative appendicular skeletal muscle mass (RASM) was obtained from the aLM/height ${ }^{2}\left(\mathrm{~kg} / \mathrm{m}^{2}\right)$ (12).

The total skeletal muscle (TSM) mass is the soft tissue mass, fat-free, bone, viscera, and internal organs. The TSM was estimated by aLM x $1.33(\mathrm{~kg})(13,14)$.

\section{Diagnosis of osteoporosis and sarcopenia}

The criteria for densitometry diagnosis of pre-sarcopenia and sarcopenia obeyed the World Health Organization (WHO) criteria and the definitions of the EWGSOP, respectively $(8,10)$. Osteoporosis was defined as femoral neck, total hip, or lumbar spine having a $\mathrm{T}$ score lower than or equal to $-2.5 \mathrm{SD}$, osteopenia was defined as a T-score more than -2.5 but less than -1.0 , and normal BMD was defined as a T-score equal to or more than -1.0. The abnormal BMD group included both osteoporosis and osteopenia subjects.

According to the EWGSOP, sarcopenia can be subclassified into three categories, depending on the stage of the disease: pre-sarcopenia (characterized by low muscle mass, without any decrease in muscle strength or physical performance); sarcopenia (defined by reduction in muscle mass accompanied by a deteriora- 
tion of strength or physical performance); and severe sarcopenia (defined by the combination of low muscle mass, physical performance and muscle strength) (8). This study did not measure severe sarcopenia.

Low muscle mass was defined as RASM (aLM/ height ${ }^{2}$ ) below a threshold of $7.26 \mathrm{~kg} / \mathrm{m}^{2}(12)$.

The muscle strength was assessed by handgrip strength. To measure the handgrip strength, a dynamometer was used (Takeikiki Kogyo, Japan, model $\mathrm{T}-2$ ) with a capacity of $100 \mathrm{kgf}$ and divisions of $\mathrm{l} \mathrm{kgf}$, adjustable and calibrated with a scale of 0 to $50 \mathrm{~kg}$. Three measurements of maximum strength were taken at both sides, and the highest value was recorded as maximal handgrip strength (8). Low muscle strength was defined as handgrip strength at $\leq 29 \mathrm{~kg}$ if $\mathrm{BMI}$ is $\leq 24, \leq 30 \mathrm{~kg}$ if BMI is $24.1-28$ and $\leq 32 \mathrm{~kg}$ if $\mathrm{BMI}$ is $>28$.

\section{STATISTICAL ANALYSIS}

Statistical analyses were performed using the SPSS statistical software (17th version; SPSS Inc., Chicago, IL).

Descriptive and association analyses showed the measurements of variables among sarcopenia diagnoses (normal, pre-sarcopenia, and sarcopenia) using analysis of variance (ANOVA) and abnormal BMD diagnosis using analysis of variance (T-Test).

Multivariable linear regression analyses with BMD as a dependent variable were performed to identify the determinants for BMD among potential factor adjustments for age and body weight. The results of all multiple linear regression analyses expressed as $\beta$ coefficients or standardized $\beta$ coefficients and $95 \%$ confidence intervals (CI).

Regression analyses were used to examine the influence of lean mass, appendicular lean mass, relative appendicular skeletal muscle mass, the total skeletal muscle, fat mass, and grip strength on abnormal BMD diagnosis. Regression analysis tested the relationship between pre-sarcopenia and sarcopenia and diagnosis of abnormal BMD with results expressed as odds ratios (OR) and 95\% CI.

\section{RESULTS}

One hundred and ninety-eight men with a mean age of 68.3 years $(S D=6.8)$, mean body weight of 68.2 $\mathrm{kg}(\mathrm{SD}=10.8)$, mean height of $1.63 \mathrm{~m}(\mathrm{SD}=0.06)$, and BMI mean of $25.44(\mathrm{SD}=3.24) \mathrm{kg} / \mathrm{m}^{2}$ were evaluated.

We found that 17 men $(8.6 \%)$ had a diagnosis of diabetes. Only one man had been diagnosed with diabetes and sarcopenia.

Only $12.6 \%$ of the patients had a diagnosis of presarcopenia and $10.1 \%$ had a diagnosis of sarcopenia.

According to table 1, we can see that men diagnosed with sarcopenia have lower BMD and T-score of the femoral neck compared to men with normal diagnosis.

Table 1. Anthropometric, grip strength and body composition analyses of normal, pre-sarcopenic and sarcopenic groups according to the definition of the EWGSOP

\begin{tabular}{|c|c|c|c|}
\hline & $\begin{array}{c}\text { Normal } \\
( \pm D P) \\
(n=153)\end{array}$ & $\begin{array}{c}\text { Pre- } \\
\text {-Sarcopenia } \\
\text { ( } \pm \text { DP) } \\
(n=25)\end{array}$ & $\begin{array}{c}\text { Sarcopenia } \\
( \pm \mathrm{DP}) \\
(\mathrm{n}=\mathbf{2 0})\end{array}$ \\
\hline Age (years) & $67^{c}$ & $67^{c}$ & $76^{a, b}$ \\
\hline Weight (kg) & $69,80 \pm 9,94^{b, c}$ & $63,10 \pm 13,22^{a}$ & $62,15 \pm 10,05^{a}$ \\
\hline Height (m) & $1,63 \pm 0,6$ & $1,64 \pm 0,06$ & $1,61 \pm 0,06$ \\
\hline BMI $\left(\mathrm{kg} / \mathrm{m}^{2}\right)$ & $26,04 \pm 3,18^{b, c}$ & $23,18 \pm 3,84^{a}$ & $23,70 \pm 3,37^{a}$ \\
\hline$\%$ fat mass & $25,45 \pm 6,71$ & $24,09 \pm 9,56$ & $25,94 \pm 9,49$ \\
\hline Total fat mass (kg) & $17,16 \pm 6,14$ & $14,26 \pm 7,24$ & $15,73 \pm 8,14$ \\
\hline Lean mass (kg) & $48,78 \pm 5,10^{\mathrm{b}, \mathrm{c}}$ & $41,89 \pm 4,20^{\mathrm{a}}$ & $41,82 \pm 3,73^{a}$ \\
\hline TSM (kg) & $29,01 \pm 3,22^{b, c}$ & $23,76 \pm 1,88^{a}$ & $23,35 \pm 2,52^{\mathrm{a}}$ \\
\hline aLM (kg) & $21,81 \pm 2,42^{\mathrm{b}, \mathrm{c}}$ & $17,87 \pm 1,41^{\mathrm{a}}$ & $17,55 \pm 1,89^{a}$ \\
\hline RASM $\left(\mathrm{kg} / \mathrm{m}^{2}\right)$ & $8,14 \pm 0,58^{\mathrm{b}, \mathrm{c}}$ & $6,60 \pm 0,52^{\mathrm{a}}$ & $6,70 \pm 0,53^{\mathrm{a}}$ \\
\hline $\begin{array}{l}\text { Grip strength } \\
\text { dominant (kgf) }\end{array}$ & $35,16 \pm 5,40^{c}$ & $34,92 \pm 4,36^{c}$ & $24,82 \pm 4,81^{a, b}$ \\
\hline $\begin{array}{l}\text { Grip strength } \\
\text { non-dominant (kgf) }\end{array}$ & $34,55 \pm 6,03^{c}$ & $33,70 \pm 4,20^{c}$ & $25,28 \pm 5,60^{a, b}$ \\
\hline $\begin{array}{l}\text { Femoral neck } \\
\text { BMD }\left(\mathrm{g} / \mathrm{cm}^{2}\right)\end{array}$ & $0,913 \pm 0,123^{c}$ & $0,862 \pm 0,118$ & $0,789 \pm 0,110^{a}$ \\
\hline $\begin{array}{l}\text { T-Score of femoral } \\
\text { neck }\end{array}$ & $-1,17 \pm 0,96 c$ & $-1,60 \pm 0,91$ & $-2,12 \pm 0,88^{a}$ \\
\hline $\begin{array}{l}\text { Total hip BMD } \\
\left(\mathrm{g} / \mathrm{cm}^{2}\right)\end{array}$ & $0,966 \pm 0,19$ & $0,908 \pm 0,13$ & $0,854 \pm 0,11$ \\
\hline T-Score of total hip & $-0,83 \pm 0,92^{\mathrm{b}, \mathrm{c}}$ & $-1,33 \pm 0,93^{a}$ & $-1,71 \pm 0,77^{\mathrm{a}}$ \\
\hline $\begin{array}{l}\text { Lumbar spine } \\
\text { BMD }\left(\mathrm{g} / \mathrm{cm}^{2}\right)\end{array}$ & $1,026 \pm 0,15$ & $0,989 \pm 0,16$ & $0,966 \pm 0,132$ \\
\hline T-Score of L1-L4 & $-1,54 \pm 1,32$ & $-1,92 \pm 1,38$ & $-2,03 \pm 1,14$ \\
\hline $\begin{array}{l}33 \% \text { radius BMD } \\
\left(\mathrm{g} / \mathrm{cm}^{2}\right)\end{array}$ & $0,736 \pm 0,82$ & $0,730 \pm 0,91$ & $0,699 \pm 0,77$ \\
\hline $\begin{array}{l}\text { T-Score of 33\% } \\
\text { radius }\end{array}$ & $-0,86 \pm 0,95$ & $-0,91 \pm 1,12$ & $-1,32 \pm 0,96$ \\
\hline
\end{tabular}

BMI: body mass index; aLM: appendicular lean mass; RASM: relative appendicular skeletal muscle mass; TSM: total skeletal muscle.

Normal, Pre-sarcopenia and sarcopenia according to the EWGSOP (15).

${ }^{a}$ ANOVA; $p<0,05=$ difference between means statistically significant in the normal group. ${ }^{\mathrm{b}} \mathrm{ANOVA} ; \mathrm{p}<0,05=$ difference between means statistically significant in the pre-sarcopenia group.

${ }^{c}$ ANOVA; $p<0,05=$ difference between means statistically significant in the sarcopenic group. 
Non-sarcopenic men have higher T-score of the total hip compared to men diagnosed with pre-sarcopenia and sarcopenia. There was no significant difference between BMD and T-score at the lumbar spine and 33\% radius among non-sarcopenic, pre-sarcopenic, and sarcopenic men.

Regarding the densitometric diagnosis, only $18.7 \%$ of the evaluated subjects had normal diagnosis. Patients with abnormal BMD (81.3\%) had significantly lower height, body weight, BMI, and grip strength in the dominant hand, lean mass, appendicular lean mass, RASM, total skeletal muscle mass, and fat mass when compared to patients with normal diagnosis. Results are shown in table 2 .

Table 2. Age, anthropometric, grip strength and body composition characteristics of normal and abnormal BMD groups

\begin{tabular}{|c|c|c|}
\hline & $\begin{array}{l}\text { Normal group } \\
\text { Mean } \pm \text { SD } \\
(n=37)\end{array}$ & $\begin{array}{c}\text { Abnormal } \\
\text { BMD group } \\
\text { Mean } \pm \text { SD } \\
(\mathrm{n}=161)\end{array}$ \\
\hline Age (years) & $67 \pm 5,9$ & $68 \pm 6,2$ \\
\hline Weight (kg) & $73,25 \pm 10,83^{*}$ & $67,02 \pm 10,46^{*}$ \\
\hline Height (m) & $1,65 \pm 0,08^{*}$ & $1,63 \pm 0,05^{\star}$ \\
\hline BMI $\left(\mathrm{kg} / \mathrm{m}^{2}\right)$ & $26,62 \pm 2,45^{*}$ & $25,17 \pm 3,34^{*}$ \\
\hline Grip strength dominant (kgf) & $35,41 \pm 6,67$ & $33,02 \pm 5,89$ \\
\hline Grip strength non-dominant (kgf) & $35,61 \pm 7,77^{\star}$ & $33,02 \pm 5,97^{*}$ \\
\hline Lean mass (kg) & $49,69 \pm 6,69^{*}$ & $46,64 \pm 5,28^{*}$ \\
\hline aLM (kg) & $22,33 \pm 3,16^{*}$ & $20,55 \pm 2,66^{*}$ \\
\hline RASM $\left(\mathrm{kg} / \mathrm{m}^{2}\right)$ & $8,11 \pm 0,63^{*}$ & $7,73 \pm 0,87^{\star}$ \\
\hline TSM (kg) & $29,71 \pm 4,20^{*}$ & $27,34 \pm 3,54^{*}$ \\
\hline Fat mass (kg) & $19,00 \pm 6,04^{*}$ & $16,11 \pm 6,56^{*}$ \\
\hline$\%$ fat & $27,22 \pm 5,98$ & $24,89 \pm 7,65$ \\
\hline
\end{tabular}

BMI: body mass index; aLM: appendicular lean mass; RASM: relative appendicular skeletal muscle mass; TSM: total skeletal muscle.

Abnormal BMD group included both osteoporosis and osteopenia subjects.

Test t; $p<0,05=$ difference between means statistically significant.
To estimate and explain the variability of BMD's femoral neck, total hip, lumbar spine, and 33\% radius by means of the variables studied, a model of linear regression, presented in table 3 , was created. The models constructed explain $98 \%$ of the variability of bone mineral densities. To select the variables that would make the model, the Stepwise method was used, and the models were adjusted for age and weight. Multiple linear regression analyses showed that relative appendicular skeletal muscle mass, lean mass, and grip strength in the dominant hand were a positive significant determinant factor for BMD in elderly men after adjusting for age and body weight.

Through logistic regression analysis, this study evaluated the influence of the variables in the diagnosis of abnormal BMD compared to patients with normal densitometric diagnosis. The results are presented in table 4.

The results of regression analyses for abnormal BMD are shown in table 4 and allow us to infer that each year, elderly men increases the chances of a diagnosis of abnormal BMD by $5.8 \%$. Together, the variables that composed the regression model for the diagnosis of abnormal BMD explained $51.7 \%$ of the variability. This result was obtained from the $\mathrm{R} 2$ model. The results of regression analyses for abnormal BMD are shown in table 4 . The study also evaluated the odds ratio of a men diagnosed with pre-sarcopenia and sarcopenia, presenting the diagnosis of abnormal $\mathrm{BMD}$. The results show that men with pre-sarcopenia are about 8 times more likely to have a diagnosis of abnormal BMD compared to normal men. Men with sarcopenia see the chance increase by 9 -fold. The results are shown in table 5 .

Table 3. Association of anthropometric, grip strength, body composition and bone density: multivariable model

\begin{tabular}{lcccc}
\hline & Femoral neck BMD & Total hip BMD & Lumbar Spine BMD & $\begin{array}{c}\text { 33\% radius BMD } \\
\text { non-dominant }\end{array}$ \\
\hline Age (years) & $0,0017(0,0000 ; 0,0033)$ & $0,0017(-0,0009 ; 0,0042)$ & $0,0074(0,0056 ; 0,0092)$ & $0,0035(0,0022 ; 0,0047)$ \\
Weight $(\mathrm{kg})$ & $0,0042(0,0024 ; 0,0060)$ & $0,0092(0,0057 ; 0,0127)$ & $0,0041(0,0019 ; 0,0063)$ & $0,0015(0,0001 ; 0,0029)$ \\
RASM $\left(\mathrm{kg} / \mathrm{m}^{2}\right)$ & $0,0632(0,0433 ; 0,0831)$ & $0,1058(0,0644 ; 0,1463)$ & - & $0,0268(0,0106 ; 0,0431)$ \\
Lean mass (kg) & - & $-0,0132(-0,0216 ;-0,0048)$ & - & - \\
Grip strength dominant (kgf) & - & - & $0,0068(0,0030 ; 0,0106)$ & $0,0053(0,0030 ; 0,0076)$ \\
$\mathrm{R}^{2}$ for the model & 0,983 & 0,967 & 0,978 & 0,987 \\
\hline
\end{tabular}

RASM: relative appendicular skeletal muscle mass.

Results expressed as $\beta$ coefficients and $95 \% \mathrm{Cl}$. Stepwise linear regression including age, weight, height, BMI: lean mass, appendicular lean mass, relative appendicular skeletal muscle mass, total skeletal muscle, fat mass and grip strength.

Adjusted for age and weight. 
Table 4. Regression analyses of body composition and muscle strength of abnormal BMD diagnosis, adjusted for age and weight

\begin{tabular}{lc}
\hline & Odds Ratio and Confidence Interval (95\%) \\
\cline { 2 - 2 } & Abnormal BMD \\
\hline Age (years) & $1,058(1,010 ; 1,108)$ \\
Weight $(\mathrm{kg})$ & $0,969(0,922 ; 1,018)$ \\
Lean mass $(\mathrm{kg})$ & $1,169(0,953 ; 1,435)$ \\
aLM $(\mathrm{kg})$ & $0,649(0,407 ; 1,034)$ \\
RASM $\left(\mathrm{kg} / \mathrm{m}^{2}\right)$ & $1,218(0,616 ; 2,408)$ \\
\hline
\end{tabular}

aLM: appendicular lean mass; RASM: relative appendicular skeletal muscle mass. Abnormal BMD defined for men with T-score $<-1,0$.

Table 5. Regression analysis of pre-sarcopenia and sarcopenia of abnormal BMD diagnosis adjusted for age and weight

\begin{tabular}{cc}
\hline & Odds Ratio (Confidence Interval - 95\%) \\
\cline { 2 - 2 } & Abnormal BMD \\
\hline Pre-sarcopenia & Reference \\
Non-sarcopenic & $8,000(3,157 ; 20,270)$ \\
Pre-sarcopenic & 0,193 \\
$\mathrm{R}^{2}$ of the model & \\
Sarcopenia & Reference \\
Non-sarcopenic & $9,000(2,088 ; 38,787)$ \\
Sarcopenic & 0,096 \\
$\mathrm{R}^{2}$ of the model &
\end{tabular}

Abnormal BMD was defined for men with T-score $<-1,0$.

Pre-sarcopenia and sarcopenia according to the EWGSOP (15).

\section{DISCUSSION}

Pre-sarcopenia and sarcopenia, characterized by a loss of muscle mass and muscle strength, are two of the main conditions responsible for the reduced functional capacity of the individual during aging, which may represent a negative influence on bone mineral density, as well as increased probability of the patient having a diagnosis of osteoporosis $(15,16)$. However, studies have shown contradicting associations, in relation to presarcopenia and sarcopenia, with BMD and diagnosis of osteoporosis $(17,18)$. Coin and cols., after evaluation of 136 men (mean age 73 years), found no significant association between BMD of the femoral neck and total hip with RASM $<7.26 \mathrm{~kg} / \mathrm{m}^{2}$, that is, diagnosed with pre-sarcopenia according to the $\operatorname{EWGSOP}(8,19)$. More recently, Verschueren and cols. found that men with pre-sarcopenia and sarcopenia were more likely to have osteoporosis compared with those with normal RASM (odds ratio 3.8; 95\% CI01.6-9.1 and 3.0; 95\% CI01.6-5.8 respectively) (4). Similarly, our results also indicate that men diagnosed with pre-sarcopenia and sarcopenia are more likely to have a higher risk of osteopenia and osteoporosis.

The association between sarcopenia and bone mass demonstrated in this study is consistent with the hypothesis of previous studies showing a reduction in osteogenic effect due to a minor mechanical stimulation imposed on the bone structure by reducing the muscle and muscle function $(4,6,15)$. Another point would be the reduction of the mechanical stimuli from less physical activity due to a lower functional capacity of the elderly caused by sarcopenia (12). However, studies to evaluate the mechanisms leading to sarcopenia to propitiate a lower bone mass are needed for more effective clinical practice for the prevention and treatment of osteoporosis in the male population. It has been estimated that the prevalence of osteoporosis in men varies between 1 and $4 \%$ of the population, and that $15-33 \%$ of men have osteopenia $(20,21)$. In contrast, in the present study sample, $33.8 \%$ of men evaluated were diagnosed with osteoporosis and $47.5 \%$ with osteopenia, totaling $81.3 \%$ of the sample with abnormal BMD. These findings may reflect greater difficulty in comparing our findings with those of other studies.

Our study found that $12.6 \%$ of the sample had a diagnosis of pre-sarcopenia and $11.9 \%$ had a diagnosis of sarcopenia. Another study showed similar results and diagnosed pre-sarcopenia in $10.1 \%$ and sarcopenia in $3.7 \%$ of the sample (4).

Our study also investigated the relationship between the components of the criteria for sarcopenia and BMD independently. We verified that the RASM and lean mass were the independent variables that best explained the variability of BMD's femoral neck, total hip, and radio $33 \%$ non-dominant. Similar findings were also observed in the study by Cui and cols. which, after evaluation of 445 men (Koreans) aged above 50 years (mean 62.7 years), found that lean mass, evaluated by DXA, was significant to explain the variability of BMD of lumbar $(b=0.271 ; p=0.004)$, femoral neck $(b=0.446 ; p$ $=0.000)$, and $33 \%$ radius $(\mathrm{b}=0.281 ; \mathrm{p}=0.001)(22)$. We also observed this relationship in the study of Kirchengast and Huber, which after evaluation of 130 men aged 60-92 years, found a significant positive association between RASM and total body BMD and femoral neck (23). These results, although not conclusive, suggest that the increase in muscle mass will provide a gain in bone mass in elderly men, which encourages the development of further studies to evaluate the effect of exercise training programs, diets, and clinical interven- 
tions directed to the increase muscle mass with the goal to prevent and treat the loss of BMD in elderly men and thus decrease the risk of osteoporotic fractures.

Another essential component for the diagnosis of sarcopenia is muscle strength. Handgrip strength is one of the muscular strength tests suggested by the EWGSOP to set the diagnosis of sarcopenia, as a method that is easy to apply, relatively inexpensive, and has a good relation with systemic muscle strength (8). In our study, we found that the higher handgrip strength in the dominant hand was associated with higher lumbar spine BMD and 33\% radius BMD, presenting a relationship as systemic as site specific with the BMD. A positive association between handgrip strength and lumbar spine BMD, may be related to osteophytes. In a study conducted with 234 men with a mean age of 47.8 years, grip strength was not shown to be a good systemic predictor of BMD, being considered nonsignificant for predicting the femoral neck and lumbar BMD after stepwise linear regression analysis (24). These results suggest that the practice of physical activities that provide increased handgrip strength can also increase the radius BMD 33\%, thus reducing the risk of wrist fractures in elderly men. New studies are needed to confirm this assumption.

Some authors evaluated the effect of fat mass on BMD in men. A study with 144 Korean men, aged 20 to 88 years, found that the mass of total body fat, appendicular fat mass, cholesterol (HDL and LDL), and triglycerides were not significant in explaining the variability of lumbar BMD (L1 - L4) (25). Similar findings were observed in the study of Kirchengast and cols. which, after evaluation of 62 men aged between 60 and 86 years, showed that fat mass, measured by DXA, was not significantly associated with BMD of the femoral neck (26). In our study, although the group of men diagnosed with abnormal BMD possessed significantly lower fat mass in relation to the group of men with normal BMD, the fat mass was not significant in predicting the variability of BMD. These findings indicate that fat mass, independently and after adjusting for body weight, has no effect on BMD, and possibly its influence is given only for their contribution in weight. More studies are needed to prove this theory.

The originality of this study lies in being the first work on the Brazilian population that used the definition proposed by the EWGSOP to distinguish sarcopenic men from normal men.
This study has several limitations. First, it is a cross sectional study. Longitudinal studies with intervention are needed to confirm whether increasing muscle mass increases BMD and a smaller risk for abnormal BMD diagnostic. Others limitations of our study would be the size and regionalization of the sample, which does not allow us to make inferences about the relationship between pre-sarcopenia, sarcopenia, and abnormal BMD in other populations. The lack of studies in the Brazilian population to verify the effectiveness of points cohort for RASM and grip strength, suggested by EWGSOP to distinguish Brazilian men with normal diagnosis from men diagnosed with pre sarcopenia and sarcopenia, may have influenced the assessment of the effect of pre-sarcopenia, sarcopenia, and the abnormal diagnostic and BMDs.

We suggest further studies, with more patients, so that the relationship between pre-sarcopenia, sarcopenia, and bone health may be better understood.

We conclude that elderly men diagnosed with presarcopenia and sarcopenia are more likely to display abnormal BMD. After adjusting for risk factors, RASM and lean mass were the independent variables that best explained the variability of BMD's. Elderly men with RASM at $<7.26 \mathrm{~kg} / \mathrm{m}^{2}$ had significantly lower BMD compared with those with RASM at $\geq 7.26 \mathrm{~kg} / \mathrm{m}^{2}$.

Our results support the need to develop programs aimed at preventing the reduction of muscle mass and strength in order to avert the reduction in bone mineral density and consequently osteoporosis.

Funding: no external funding, apart from the support of the authors' institution was available for this study.

Author contributions: all authors have played a role in the conception, development, planning, execution, analysis or interpretation of the research and/or writing of the manuscript and they all agree and accept responsibility for the contents of the manuscript submitted to ABEM. All authors have read and approved the submitted manuscript.

Disclosure: no potential conflict of interest relevant to this article was reported.

\section{REFERENCES}

1. Melton LJ 3rd, Gabriel SE, Crowson CS, Tosteson AN, Johnell O, Kanis JA. Cost-equivalence of different osteoporotic fractures. Osteoporos Int. 2003;14(5):383-8.

2. Burge $R$, Dawson-Hughes $B$, Solomon DH, Wong JB, King A, Tosteson A. Incidence and economic burden of osteoporosisrelated fractures in the United States, 2005-2025. J Bone Miner Res. 2007;22(3):465-75. 
3. Szulc P, Beck TJ, Marchand F, Delmas PD. Low skeletal muscle mass is associated with poor structural parameters of bone and impaired balance in elderly men-the MINOS study. J Bone Miner Res. 2005;20(5):721-9.

4. Verschueren S, Gielen E, O'Neill TW, Pye SR, Adams JE, Ward $K A$, et al. Sarcopenia and its relationship with bone mineral density in middle-aged and elderly European men. Osteoporos Int. 2013;24(1):87-98.

5. Genaro PS, Pereira GA, Pinheiro MM, Szejnfeld VL, Martini LA. Influence of body composition on bone mass in postmenopausal osteoporotic women. Arch Gerontol Geriatr. 2010;51(3):295-8.

6. Rochefort GY, Pallu S, Benhamou CL. Osteocyte: the unrecognized side of bone tissue. Osteoporos Int. 2010;21(9):1457-69.

7. Di Monaco M, Vallero F, Di Monaco R, Tappero R. Prevalence of sarcopenia and its association with osteoporosis in 313 older women following a hip fracture. Arch Gerontol Geriatr. 2011;52(1):71-4.

8. Cruz-Jentoft AJ, Baeyens JP, Bauer JM, Boirie Y, Cederholm T, Landi F, et al. Sarcopenia: European consensus on definition and diagnosis: report of the European Working Group on Sarcopenia in Older People. Age Ageing. 2010;39:412-23.

9. Bijlsma $A Y$, Meskers CG, Ling $\mathrm{CH}$, Narici M, Kurrle SE, Cameron ID, et al. Defining sarcopenia: the impact of different diagnostic criteria on the prevalence of sarcopenia in a large middle aged cohort. Age (Dordr). 2013;35(3):871-81.

10. WHO. Assessment of fracture risk and its application to screening for postmenopausal osteoporosis. WHO Study Group. World Health Organ Tech Rep Ser. 1994;843:1-129.

11. Heymsfield SB, Smith R, Aulet M, Bensen B, Lichtman S, Wang J, et al. Appendicular skeletal muscle mass: measurement by dualphoton absorptiometry. Am J Clin Nutr. 1990;52(2):214-8.

12. Baumgartner RN, Koehler KM, Gallagher D, Romero L, Heymsfield SB, Ross RR, et al. Epidemiology of sarcopenia among the elderly in New Mexico. Am J Epidemiol. 1998;147(8):755-63.

13. Wang ZM, Visser M, Ma R, Baumgartner RN, Kotler D, Gallagher $D$, et al. Skeletal muscle mass: evaluation of neutron activation and dual-energy x-ray absorptiometry methods. J Appl Physiol. 1996;80(3):824-31.

14. Iannuzzi-Sucich M, Prestwood KM, Kenny AM. Prevalence of sarcopenia and predictors of skeletal muscle mass in healthy, older men and women. J Gerontol A Biol Sci Med Sci. 2002;57(12):772-7.
15. Marcell TJ. Sarcopenia: causes, consequences, and preventions. J Gerontol A Biol Sci Med Sci. 2003;58(10):911-6.

16. Blain $H$, Jaussent $A$, Thomas $E$, Micallef JP, Dupuy AM, Bernard $\mathrm{PL}$, et al. Appendicular skel- etal muscle mass is the strongest independent factor associated with femoral neck bone mineral density in adult and older men. Exp Gerontol. 2010;45(9):679-84.

17. Wu CH, Yang KC, Chang HH, Yen JF, Tsai KS, Huang KC. Sarcopenia is related to increased risk for low bone mineral density. J Clin Densitom. 2013;16(1):98-103.

18. Rondanelli M, Guido D, Opizzi A, Faliva MA, Perna S, Grassi M. A path model of sarcopenia on bone mass loss in elderly subjects. J Nutr Health Aging. 2014;18(1):15-21.

19. Coin A, Perissinotto E, Enzi G, Zamboni M, Inelmen EM, Frigo AC, et al. Predictors of low bone mineral density in the elderly: the role of dietary intake, nutritional status and sarcopenia. Eur $\mathrm{J}$ Clin Nutr. 2008;62:802-9.

20. Looker AC, Johnston CC, Wahner HW, Dunn WL, Calvo MS, Harris TB, et al. Prevalence of low femoral bone density in older U.S. women from NHANES III. J Bone Miner Res. 1995;10:796-802.

21. Lambert JK, Zaidi M, Mechanick JI. Male osteoporosis: epidemiology and the pathogenesis of aging bones. Curr Osteoporos Rep. 2011;9(4):229-36.

22. Cui LH, Shin MH, Kweon SS, Park KS, Lee YH, Chung EK, et al. Relative contribution of body composition to bone mineral density at different sites in men and women of South Korea. J Bone Miner Metab. 2007;25(3):165-71.

23. Kirchengast S, Huber J. Sex-specific associations between soft tissue body composition and bone mineral density among older adults. Ann Hum Biol. 2012;39(3):206-13.

24. Aydin G, Atalar E, Keles I, Tosun A, Zog G, Keles H, et al. Predictive value of grip strength for bone mineral density in males: site specific or systemic? Rheumatol Int. 2006;27(2):125-9.

25. Yoo HJ, Park MS, Yang SJ, Kim TN, Lim KI, Kang HJ, et al. The differential relationship between fat mass and bone mineral density by gender and menopausal status. J Bone Miner Metab. 2012;30(1):47-53.

26. Kirchengast S, Peterson B, Hauser G, Knogler W. Body composition characteristics are associated with the bone density of the proximal femur end in middle- and old-aged women and men. Maturitas. 2001;39:133-45. 